\title{
Postmortem histological survey of the ocular lesions in a British population of AIDS patients
}

\author{
I Pecorella, A Ciardi, A Garner, A C E McCartney, S Lucas
}

\begin{abstract}
Aims-To study ocular pathology and systemic correlations in a series of 73 postmortem eyes from British patients who died from AIDS before the introduction of a HAART regimen.

Methods-The eyes were studied with conventional histology, special histochemical stainings, and immunohistochemistry.

Results-72.6\% of the cases showed chronic uveal inflammation, caused by opportunistic agents in $37.7 \%$ of them (cytomegalovirus (CMV) in $30.1 \%, C$ neoformans in $5.6 \%$, and Gram positive bacteria in $1.8 \%$ ). Cytoid bodies were noted in 10/73 eyes, three linked to CMV retinitis. Six retinal haemorrhages, four of which were secondary to CMV, were found. 14 specimens $(19.1 \%)$ showed foci of calcification, and a further 11 (15\%) calcium oxalate deposits. In no cases were the calcific deposits suspected clinically. Six eyes $(8.2 \%)$ did not show any abnormality.

Conclusions-CMV retinitis is the most common $(28.7 \%, 21 / 73)$ ocular infection in this series and may occur either during or in the absence of systemic dissemination. Conversely, ocular cryptococcosis appears to be an epiphenomenon of systemic and CNS disease. No other opportunistic ocular infections were present in this series. Interesting findings were the presence of intraocular precipitates of calcium oxalate and calcium phosphate or carbonate in a significant number of cases $(15 \%$ and $19 \%$, respectively), and the high prevalence of idiopathic uveal inflammation $(43.8 \%)$.

(Brf Ophthalmol 2000;84:1275-1281)
\end{abstract}

Ocular involvement in the acquired immunodeficiency syndrome (AIDS) is widely recognised. The introduction of a highly active antiretroviral regimen (HAART) has not only improved survival of AIDS patients, but has induced a dramatic downturn in the number of cases of cytomegalovirus (CMV) retinitis ${ }^{1}$ and other ocular infections. Before the HAART era, most commonly, ocular lesions developed late in the course of the infection, with the effect that the increased survival rates resulting from improved therapeutic measures were accompanied by an increased incidence of ocular disease. ${ }^{2}$ The prevalence of different populations at risk for AIDS varies between countries, as does the endemicity of the opportunist pathogens responsible for many of its ocular complications, which means that inci- dence rates from different centres are not necessarily comparable. Moreover, variations in clinical diagnostic criteria can add a further degree of uncertainty.

To date there are only a few histopathological studies of the postmortem ocular findings in AIDS patients, ${ }^{3-6}$ and only two of them ${ }^{35}$ correlate these findings with the systemic involvement of infections or tumours. We now present findings at postmortem examination in a British population composed exclusively of homosexual or bisexual males. We believe that a documented clinicopathological study of the ocular lesions observed in a patient population before the HAART era might help in the comparison of the two groups of patients and provide a baseline for measuring the efficacy of treatments. Furthermore, because our series is composed exclusively of homosexual individuals, the data we provide are different from other histological studies from other countries with a different prevalence of risk factors.

\section{Material and methods}

Seventy three subjects with clinically and immunologically confirmed AIDS underwent a full postmortem examination between 1989 and 1994 at University College Hospital, London with histopathological study of one eye (in two instances both eyes were processed). The eyes were selected according to either a clinical history of ocular disease or randomly, when such evidence could not be obtained. The eyes and a short segment of the intraorbital portion of the optic nerve were fixed in formalin, before being opened horizontally at the superior edge of the cornea and examined macroscopically with a dissecting microscope. Unless the calottes of the eyes appeared to be macroscopically abnormal, only the central portions of the eyeballs were processed to paraffin embedding, and sectioned at three or more levels before staining with haematoxylin and eosin, periodic acid Schiff, Gram stain, mucicarmine, ZiehlNeelsen, Warthin-Starry, and Gomori's methenamine stain. In cases with microscopic features consistent with a possible calcium salt

Table 1 Non-infectious and non-neoplastic histological ocular features in our series of AIDS patients

\begin{tabular}{lll}
\hline Feature & No of patients & $\%$ \\
\hline Idiopathic uveitis & 32 & 43.8 \\
Cytoid bodies & 10 & 13.6 \\
Retinal haemorrhage & 6 & 8.2 \\
Subconjunctival haemorrhage & 1 & 1.3 \\
Thrombi & 2 & 2.7 \\
Retinal coagulative necrosis & 2 & 2.7 \\
Calcium deposits & 14 & 19.1 \\
Oxalate deposits & 11 & 15 \\
Pinguecula & 8 & 10.9 \\
Choroidal naevus & 3 & 4.1 \\
No abnormality & 6 & 8.2 \\
\hline
\end{tabular}




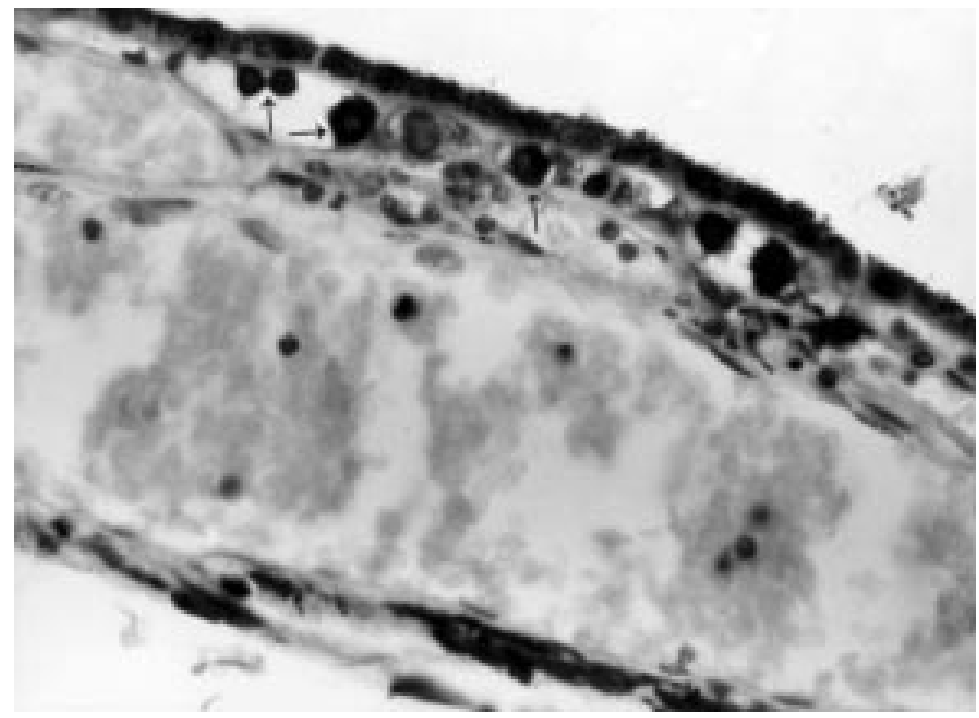

Figure 1 Note multiple cryptococci (arrows) in the lumina of the vessels in the choriocapillaris. The organisms are stained dark red with mucicarmine and this allows us to distinguish $C$ neoformans from Histoplasma (mucicarmine, $\times 400$ ).
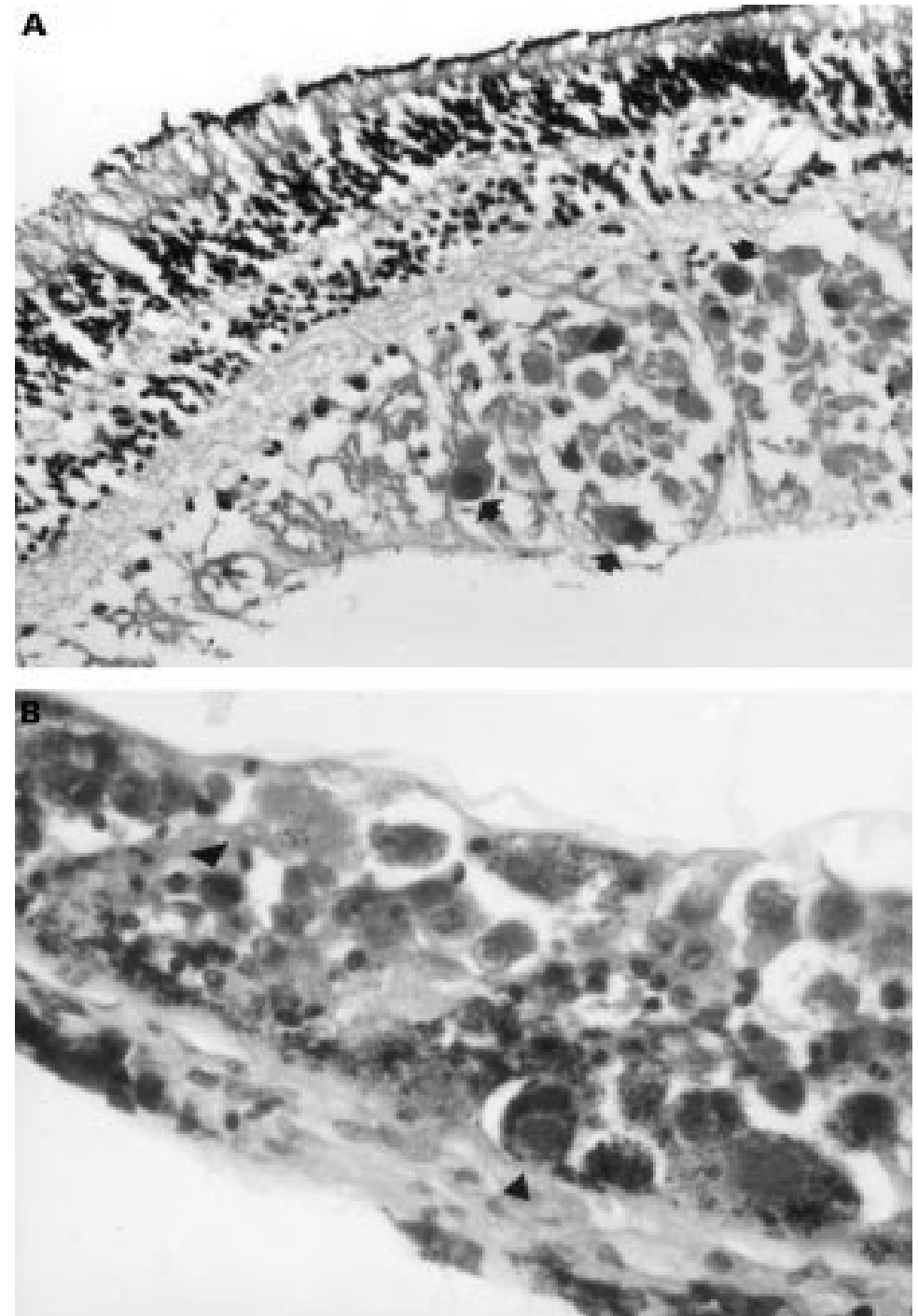

Figure 2 Histologically, cytoid bodies (A) (arrows) can resemble CMV infected cytomegalic cells (B) (arrowheads). However, the latter have a clear halo around the nuclear viral inclusion and the cytoplasmic borders are often recognisable (haematoxylin and eosin, $\times 250$ ). deposition, von Kossa's and Yasue's stains for calcium phosphate and oxalate, respectively, were also performed. Immunohistochemistry, using a standard biotin-streptavidinperoxidase technique, was used in all of the cases to demonstrate the presence of HIV p24 antigen (Dupont), whereas searches for Toxoplasma gondii (Biogenex), herpes simplex virus types 1 and 2 (Dako) and paramyxovirus (antibody kindly donated by Dr A Warnes), and in situ hybridisation with a probe for cytomegalovirus (CMV) (Enzo Diagnostics) and for varicella zoster virus (Keratech Biotechnology BV) were undertaken in cases with consistent histological findings. In five instances transmission electron microscopy was performed.

All the 73 patients were male and homosexual (two bisexual) with overt AIDS, while one was also an injecting drug user. Five of the subjects originated from Barbados, Jamaica, and Trinidad (one each), and Spain (two). Apart from those coming from the West Indies, all the cases were white. Ages ranged from 26 to 59 years (mean 37.8 years). The notes were available for 47 patients and were reviewed retrospectively with regard to the ophthalmological findings in the last year of life.

\section{Results}

HISTOLOGICAL FINDINGS

In the two instances in which both eyes were examined no difference was detected on the two sides and the results are presented with respect to the number of cases rather than the number of eyes examined.

The most frequent finding was uveal inflammation in 53 of the 73 cases $(72.6 \%)$. In seven it was confined to the anterior uvea, in 12 to the choroid, while in 34 it involved the entire uvea. Generally, the inflammation was of mild or moderate severity, showing focal aggregates of mononuclear cells in the uveal interstitium, but in four there was a severe panuveitis. The inflammation appeared to be secondary to CMV infection in 16 instances, to Cryptococcus neoformans infection in three, and Gram positive bacillary infection in one. Although one patient with severe panuveitis and associated necrosis of the entire retina and acute keratitis had had a history of shingles affecting the ophthalmic branch of the trigeminal nerve and causing ophthalmic arteritis, no aetiological agent could be demonstrated in the remaining 32 cases (Table 1). Five cases of retinal necrosis (retinitis) attributable to CMV infection were identified in the absence of uveitis. Cryptococcal infection was invariably mild with occasional organisms located next to the choriocapillaris (Fig 1) and rarely in the retina: a mild to moderate granulomatous uveal response in the site of cryptococcal invasion was observed in three cases, but retinal inflammation was not seen. Special stains in these three cases detected no other associated intraocular infections. Solitary cryptococcal involvement of the optic nerve meninges in the absence of inflammation was noted in one instance. A few yeasts suggestive of Histoplasma capsulatum were seen in the ciliary body and 


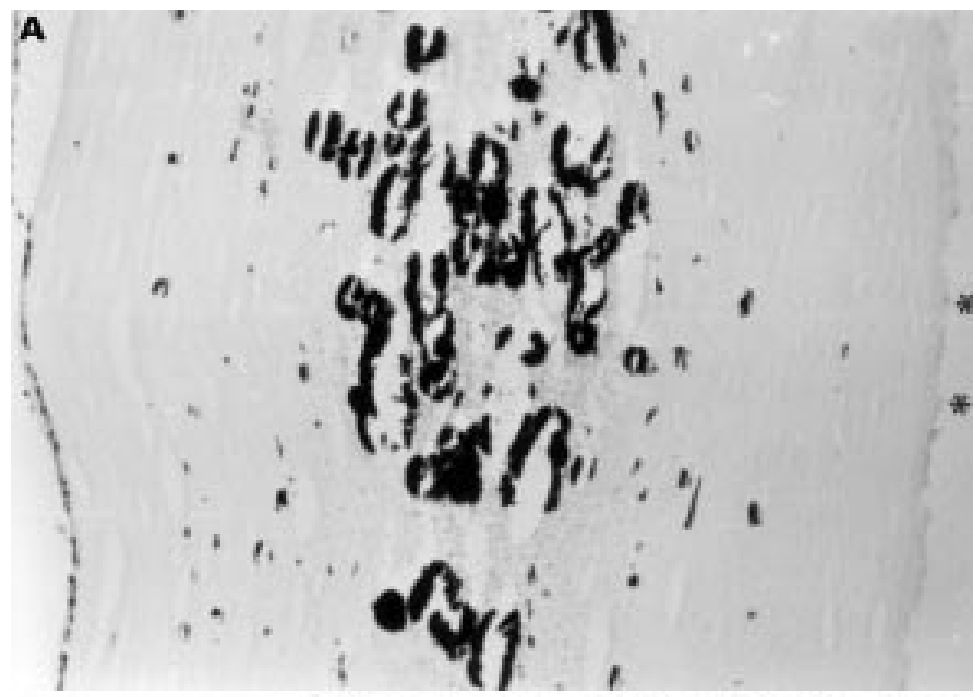

B

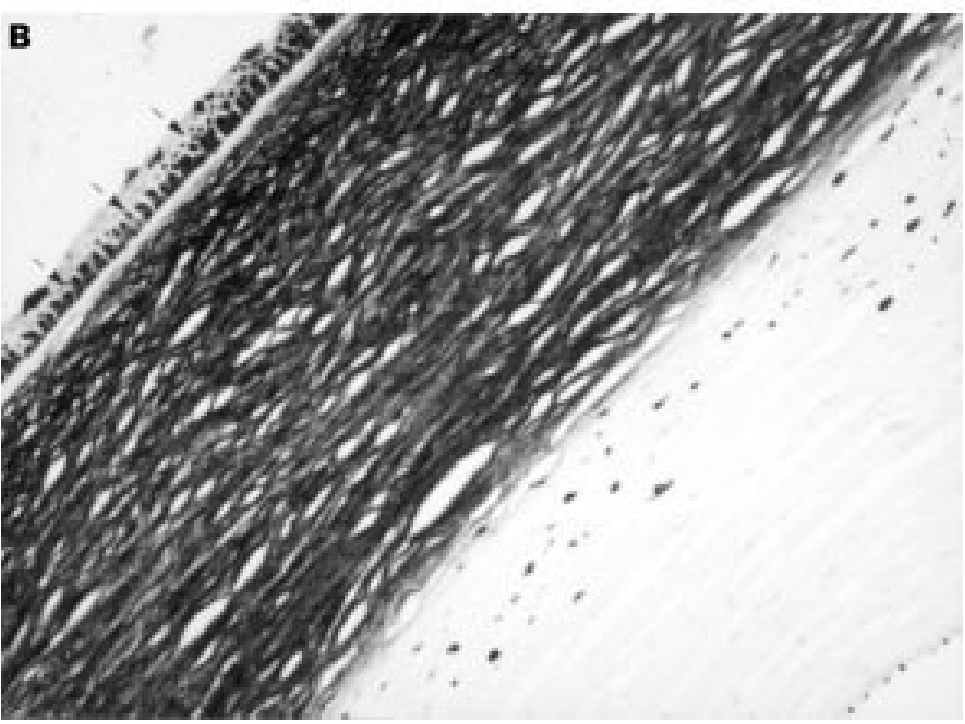

Figure 3 Two examples of corneal calcification: histology disclosed a rather localised bilateral mid stromal deposit in this 34 year old bisexual AIDS patient. The corneal epithelium (asterisks) is artefactually lost $(A)$. Calcification was more superficial and diffuse in another 44 year old homosexual individual, and included focal groups of basal epithelial cells (arrows). Other deposits were observed in the sclera, optic nerve body, and iridociliary pigmented epithelium of this patient (von Kossa's stain, $\times 250$ and $\times 25$, respectively). necrosis of the retina without demonstrable circulatory deficiency (Table 1 ).

Ocular calcification was a feature of 14 eyes $(19.1 \%)$, affecting the stroma of the ciliary processes in eight instances, the sclera in five, the peripheral choroidal blood vessels in two, the cornea in three (Fig 3A, B), the bulbar and palpebral conjunctiva in one, Bruch's membrane in one, the optic nerve body and an extraocular muscle in another one. A giant drüsen of the optic nerve head and calcified drüsens of the peripheral retinal pigment epithelium were also observed. The deposits were amorphous, basophilic, and stained with von Kossa's method. Also observed were 11 eyes $(15 \%)$ with focal or more widespread greyish crystals located in the walls of the retinal blood vessels and deposited on the inner limiting lamina of the retina. Similar deposits were also seen in the retinal pigment epithelium of one case, along the surface of the ciliary processes and the ciliary body in two cases, and in another they involved the sclera and anterior orbital soft tissues and palpebral blood vessels. On the basis of polarising microscopy and Yasue's staining method the crystals were identified as calcium oxalate (Fig 4A, B). Of the 24 eyes with calcific deposits, 18 showed other microscopic abnormalities (see Table 2).

Other miscellaneous findings included pingueculae in eight cases, elastotic degeneration of the forniceal and palpebral conjunctiva in one, and benign choroidal melanocytic naevi in three. These lesions are possibly purely coincidental, although the finding of stromal elastosis in an unexposed region of the conjunctiva is unusual and pinguecula is relatively rare in Britain. ${ }^{7}$ Six eyes $(8.2 \%)$ failed to show any abnormality (Table 1 ).

CLINICOPATHOLOGICAL CORRELATIONS

Thirty nine of the 47 patients whose notes were reviewed had had an ophthalmological examination in the year before their death.

The clinical notes were available for 18 of the 32 patients diagnosed histologically as having idiopathic uveal inflammation. However, there was no objective abnormality in 15 patients who were examined ophthalmoscopically: the remaining three had no clinical complaint and were not examined. It should be noted that only eight of the 18 patients had been examined in the last month of life. Of the two patients with severe idiopathic panuveitis, one was blind and had associated evidence of extensive retinal necrosis; the other was reported as having a normal fundus when examined 1 month before death, but subsequent histological study revealed a calcific giant drüsen of the optic disc, a calcified posterior scleral plaque, and focal calcification of the optic nerve and an extraocular muscle, as well as elastotic degeneration of the palpebral conjunctiva. The causes of death for patients with idiopathic uveal inflammation are listed in Table 3.

The histological findings confirmed the presence of CMV retinitis in 14 patients diagnosed antemortem and also showed that there were small foci of activity in two cases consid- body of two cases $(2.7 \%)$ without CMV infection and another two showed focal coagulative 

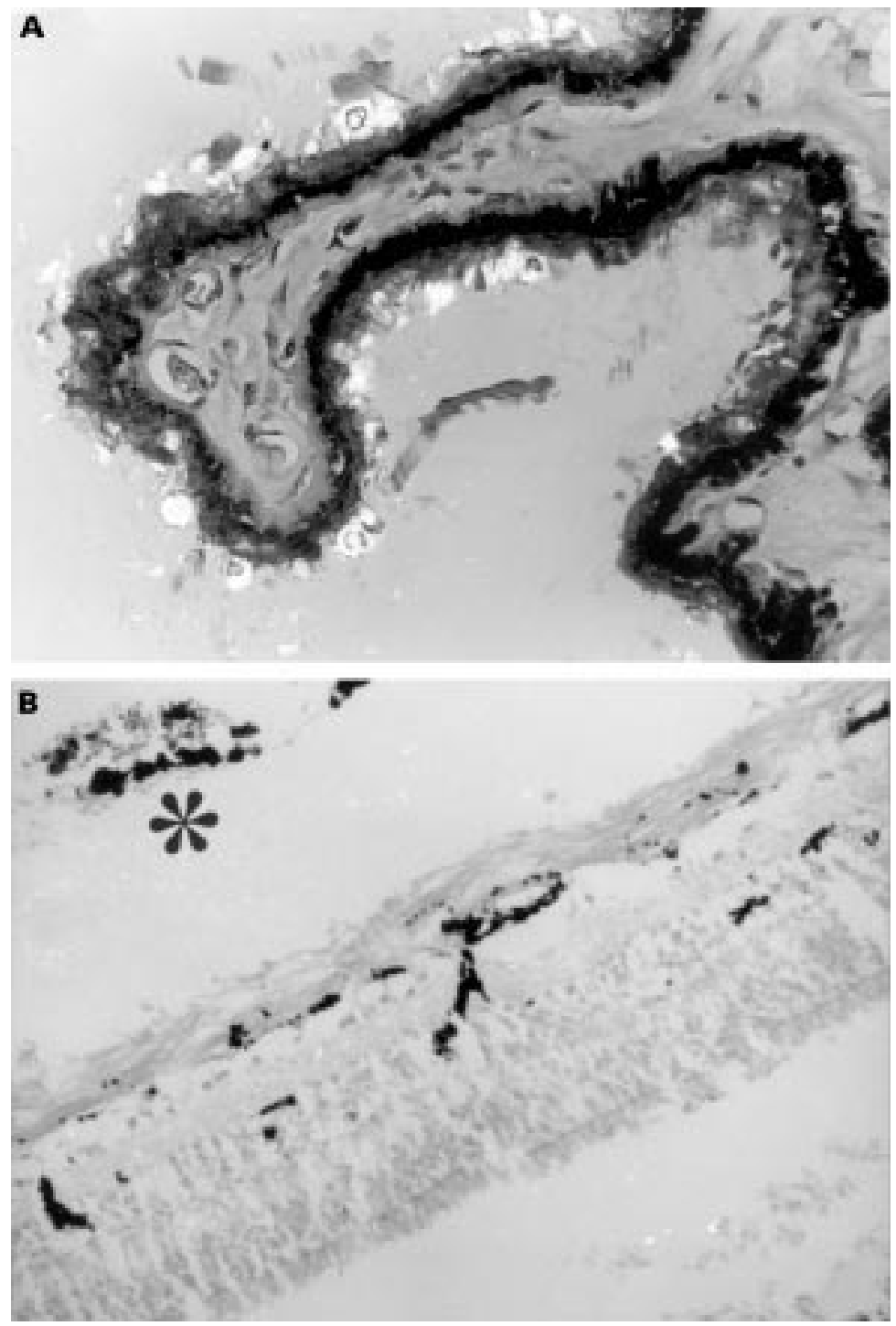

Figure 4 Oxalate crystals were deposited mainly on basal membranes. In $(A)$, the oxalates outline the contour of the ciliary processes $(\times 100$, crossed polarised light $)$. In $(B)$, they involve the wall of the retinal blood vessels. Deposits are also seen in the vitreal cavity (asterisk), next to the nerve fibre layer (Yasue's stain, $\times 25$ ).

ered to be clinically quiescent. Of the remaining seven patients with microscopic CMV retinitis, one had not been examined, one had been seen before death and the fundi not visualised, and another one with only choroidal CMV infection showed no abnormalities at funduscopy. Four patients had no clinical notes available.

No abnormality was recorded in the three patients with histological evidence of corneal calcification when they were examined 1 month before death.

The notes of seven of the 11 cases with posterior segment calcium oxalate deposition were available: five had been examined ophthalmoscopically, and three of them on the last admission, but in none was oxalosis suspected.

OCULAR AND SYSTEMIC CORRELATIONS

The overall postmortem histology of the 73 patients was reviewed and data relating to the ocular histology in those with identified systemic disease or with non-ocular neoplasms are detailed in Table 4

Systemic CMV infection was recognised in $42(57.5 \%)$ cases and of these 15 had associated ocular involvement. Thirty two patients had terminal bacterial infections, seven having septicaemia, but ocular pyogenic bacterial disease (necrotising iritis) was observed in only one instance. Four of five patients with disseminated cryptococcosis had ocular or optic nerve sheath involvement. Two patients had herpetic ulcers of the upper respiratory tract and skin, respectively, but in neither case were the eyes involved. Other systemic infections were candidiasis (23 cases), aspergillosis (two cases), histoplasmosis (one case), mycobacteriosis (19 cases), pulmonary and extrapulmonary pneumocystosis (18 cases), and cerebral toxoplasmosis (one case). In none of these were the eyes implicated.

Twenty five patients died with Kaposi's sarcoma: 20 had cutaneous lesions, including two with eyelid involvement. No conjunctival tumours were seen although one patient had a subconjunctival mass which histology showed to be a large haemorrhage. Ten patients developed lymphomas but without ocular or orbital involvement.

\section{Discussion}

This is the largest European histological study performed on eyes removed at necropsy from AIDS patients and includes 73 British cases with complete postmortem and clinical data. The benefit of histopathological examination is that it adds to the reliability of purely clinical diagnosis, but at the time of writing only few other such series have been documented. Two from the $\mathrm{USA}^{58}$ described the ocular findings at post mortem in 35 and 206 patients, respectively, and another three were reports of 25 French, ${ }^{3} 43$ Danish, ${ }^{4}$ and 48 Swedish $^{2}$ cases. However, three of these studies ${ }^{248}$ do not present full data on the systemic findings in their patients.

In respect of CMV retinitis, cryptococcosis, and toxoplasmosis, the incidence of ocular infection by the various pathogens encountered in the three complete series, including the present one, reflects the incidence of systemic involvement, albeit at lower levels (Table 4). Thus, a $57.5 \%$ incidence of systemic CMV infection in the present study was associated with a $20.5 \%$ retinal involvement, which compares with the corresponding figures of $71.4 \%$ and $34.2 \%$, respectively, in the American series ${ }^{5}$ and of $36 \%$ and $20 \%$, respectively, in the French study. ${ }^{3}$ In all three series CMV retinitis was much the most common ocular infection in AIDS sufferers and, as in three $(12 \%)$ of the French patients, in six of our cases $(8.2 \%)$ it was apparently confined to the eye. The prevalence of CMV retinitis in homosexual men does not appear to differ from other series of patients with different risk factors, as shown by other studies where a prevalence of $28 \%{ }^{9}$ and $31 \%{ }^{10}$ were recorded.

By contrast, ocular cryptococcosis in AIDS patients was never seen in either the present series or that from the USA in the absence of 
Table 2 Sites of calcium (Ca) deposition and associated ocular microscopical abnormalities

\begin{tabular}{lll}
\hline Ca hydroxyapatite & & \\
\hline Case & Sites & Associated abnormalities \\
\hline 1 & C, S, CG & Mild posterior uveitis, pinguecula \\
2 & C, CP, CV & Moderate panuveitis, CWS \\
3 & C & - \\
4 & S, CP & Mild poosterior uveitis \\
5 & S & Severe panuveitis \\
6 & S, ON & Moderate panuveitis \\
7 & S & Mild panuveitis \\
8 & CP & Mild panuveitis and cheratitis \\
9 & $\mathrm{CP}$ & CMV chorioretinitis \\
10 & $\mathrm{CP}, \mathrm{R}$ & - \\
11 & $\mathrm{CP}, \mathrm{CV}$ & Mild panuveitis, CWS \\
12 & $\mathrm{BM}$ & RV oxalosis \\
13 & $\mathrm{CP}$ & \\
14 & & \\
\hline
\end{tabular}

\section{Ca oxalate}

\begin{tabular}{lll}
\hline 1 & RV, NFL & CMV chorioretinitis \\
2 & RV, NFL & CWS \\
3 & RV, VRI & Mild anterior uveitis and cheratitis \\
4 & VRI & Mild panuveitis \\
5 & Widespread & Moderate panuveitis, \\
6 & RV, VRI & Moderate panuveitis, \\
7 & RV, RPE & Moderate panuveitis, \\
8 & Widespread & CMV chorioretinitis \\
9 & EV & - \\
10 & EV & -
\end{tabular}

$\mathrm{C}=$ cornea, $\mathrm{S}=$ sclera, $\mathrm{CG}=$ conjunctiva, $\mathrm{R}=$ retina, $\mathrm{CP}=$ ciliar processes, $\mathrm{CV}=$ choroidal blood vessels, $\mathrm{BM}=$ Bruch's membrane, $\mathrm{ON}=$ optic nerve, $\mathrm{EM}=$ extraocular muscles, $\mathrm{RV}=$ retinal blood vessels, $\mathrm{EV}=$ eyelid blood vessels, $\mathrm{HSV}=$ herpes simplex virus, NFL = nerve fibre layer, $\mathrm{VRI}=$ vitreoretinal interface, $\mathrm{RPE}=$ retinal pigmented epithelium, $\mathrm{CWS}=$ cotton wool spots.

Table 3 Postmortem findings in patients with uveitis of unknown origin

\begin{tabular}{|c|c|c|c|}
\hline Case & Uveitis & Grade & Causes of death \\
\hline 1 & Pan & 1 & PCP, MAI, cardiomiopathy \\
\hline 2 & Ant & 1 & PCP, mild CMV \\
\hline 3 & Pan & 3 & KS, HIVE, CMV and bacterial pneumonia \\
\hline 4 & Post & 2 & Diss-CMV, KS, bacterial pneumonia, toxoplasmosis \\
\hline 5 & Ant & 1 & Cerebral death \\
\hline 6 & Post & 1 & PCP, KS, CMV adrenalitis \\
\hline 7 & Pan & 2 & PCP, bacterial penumonia, mild CMV, HIVE \\
\hline 8 & Pan & 1 & Diss-CMV, bacterial penumonia, diss-KS, CMVE \\
\hline 9 & Pan & 1 & Pulmonary oedema, cerebral infarct \\
\hline 10 & Pan & 1 & Diss-CMV, KS, HIVE \\
\hline 11 & Ant & 1 & Diss-CMV, lymph node and brain lymphoma, PCP \\
\hline 12 & Ant & 1 & Diss-CMV, lung KS and aspergillosis; cryptosporidiosis \\
\hline 13 & Ant & 1 & $\begin{array}{l}\text { Haemorrhagic contusion of brain, diss-CMV, bacterial } \\
\text { pneumonia }\end{array}$ \\
\hline 14 & Pan & 1 & $\begin{array}{l}\text { MAI spleen and nodes, Gram+ septicaemia, cryptosporidiosis, } \\
\text { diss-KS, CMV colitis, CMVE }\end{array}$ \\
\hline 15 & Pan & 2 & Diss-KS, mild CMV \\
\hline 16 & Pan & 1 & Pyopneumothorax, sclerosing cholangitis \\
\hline 17 & Pan & 1 & $\mathrm{CMV}$ and $\mathrm{PC}$ pneumonia, CMV brain \\
\hline 18 & Ant & 1 & PCP \\
\hline 19 & Post & 1 & Diss-CMV, cerebral lymphoma, HIVE \\
\hline 20 & Pan & 1 & Bacterial pneumonia, ATN, MAI nodes, CMV brain, VM \\
\hline 21 & Pan & 2 & $\begin{array}{l}\text { KS, histoplasmosis, nodal MAI, ATN, spontaneous cerebral } \\
\text { haemorrhage }\end{array}$ \\
\hline 22 & Pan & 2 & Hodgkin's lymphoma, ATN \\
\hline 23 & Post & 1 & PCP \\
\hline 24 & Ant & 1 & PCP, TBC, HIVE \\
\hline 25 & Pan & 1 & $C \operatorname{spp}$ Pneumonia, ATN, partially treated TBC, VM \\
\hline 26 & Pan & 2 & $\begin{array}{l}\text { Myelodysplasia, } C s p p \text { and interstitial pneumonia, ATN, KS, } \\
\text { mild CMV }\end{array}$ \\
\hline 27 & Post & 1 & $\begin{array}{l}\text { PCP, VM, multiple thrombi with brain, spleen and kidney } \\
\text { infarction }\end{array}$ \\
\hline 28 & Pan & 1 & Hepato-renal failure, diss-CMV, KS, cerebral microinfarctions \\
\hline 29 & Pan & 1 & Bacterial pneumonia, PML, VM, HIVE \\
\hline 30 & Pan & 2 & Bacterial pneumonia diss-CMV, nodal KS \\
\hline 31 & Pan & 2 & Bacterial pneumonia, myocarditis, cerebral microinfarcts \\
\hline 32 & Post & 1 & Cirrhosis, visceral CMV, ATN \\
\hline
\end{tabular}

$\mathrm{Ant}=$ anterior; $\mathrm{ATN}=$ acute tubular necrosis, $C \mathrm{spp}=$ Candid $a$ species $; \mathrm{CMVE}=\mathrm{CMV}$ encephalitis; Diss-CMV = disseminated CMV; HIVE = HIV encephalitis; KS = Kaposi's sarcoma MAI = Mycobacterium avium intracellulare $; \mathrm{Pan}=$ panuveitis $; \mathrm{PCP}=$ Pneumocystis carinii pneumonia PML = progressive multifocal leucoencephalopathy; Post = posterior; TBC = tuberculosis; VM = vacuolar myelopathy.

systemic or CNS involvement, which suggests that the eye is unlikely to be a primary infection site. However, relative to the levels of ocular infection accompanying systemic candidiasis, aspergillosis, and other mycoses there may be an enhanced risk that AIDS patients with systemic cryptococcal infection will succumb to ocular complications (four out of five cases in the present study and two out of five cases in the American series: an overall incidence between the three series of $50 \%$ ). The low level of ocular cryptococcosis in AIDS patients as a whole (six of 133 cases (4.5\%)) appears to be a reflection of the prevalence of systemic infection. Although $C$ neoformans has been shown to destroy the anterior visual pathways but only produce minimal inflammation in the process, $^{11}$ in our three cases with intraocular cryptococcal infection and no other microorganism detectable at the special stains, a mild to moderate choroiditis was observed.

Rare instances of ocular candidiasis in association with AIDS have been recorded ${ }^{12}$ and infection appears to be more likely in injecting drug user with candidaemia. The low number of injecting drug users in our series probably explains the absence of ocular infection by Candida or Aspergillus spp, despite the moderately high incidence of systemic infection of the former in both the British and American studies. There are a few well documented reports of ocular histoplasmosis ${ }^{13}$ and, contrary to our experience, of pneumocystosis ${ }^{14}$, toxoplasmosis, ${ }^{12}{ }^{15}$ tuberculosis, ${ }^{16}$ and Mycobacterium avium intracellulare choroiditis ${ }^{17}$ in AIDS patients in the literature. They are, nevertheless, as all three postmortem studies suggest, uncommon infections. The case of histoplasmosis reported by Specht ${ }^{13}$ indicated a long delay between systemic and ocular infections and, given the rapid death of some AIDS patients, this may possibly contribute to a lowered incidence of ocular involvement. It may be relevant that one of our patients who eventually died from disseminated histoplasmosis had only a very few yeasts in the choroid with minimal evidence of an inflammatory response. Infection in the solitary case of toxoplasmosis in our series was confined to the brain, thus resembling the American experience, ${ }^{5}$ and although the French study $^{3}$ revealed a much higher incidence of cerebral involvement, there was only one case in which the eyes were affected. This is interesting since a retinochoroiditis incidence of $3 \%$ is cited in respect of AIDS sufferers on clinical grounds. ${ }^{14}$ Possibly the reliability of such diagnoses should be questioned..$^{15}$ Moreover, a large neuropathological study of 269 AIDS cases revealed CNS toxoplasmosis in just $2.6 \%,{ }^{18}$ which taken with the observation that only $10-20 \%$ of those who have intracranial disease can be expected to incur ocular infection, ${ }^{10}$ suggests that the true incidence is considerably lower. The absence of ocular pneumocystis infection in any of the three histopathological series despite a moderately high level of pulmonary involvement may reflect the infrequency of extrapulmonary dissemination overall (demonstrated in only three of the 18 patients with lung infection in the present series). It should be noted that several of our patients had been treated with prophylactic aerosolised pentamidine therapy. Nevertheless, even in patients with 
Table 4 Histologically proved systemic infections and neoplasms (\%) and concomitant ocular involvement in our series compared to two other studies

\begin{tabular}{|c|c|c|c|c|c|c|c|c|c|}
\hline \multirow[b]{2}{*}{ Pathology } & \multicolumn{3}{|c|}{ This study (73 patients) } & \multicolumn{3}{|c|}{ Pepose (35 patients) } & \multicolumn{3}{|c|}{ De Girolami (25 patients) } \\
\hline & $S$ & $S+E$ & $E$ & $S$ & $S+E$ & $E$ & $S$ & $S+E$ & $E$ \\
\hline P carinii & $18(24.6)$ & - & - & $26(74)$ & - & - & $18(72)$ & - & - \\
\hline $\mathrm{CMV}^{1}$ & $42(57.5)$ & $15(20.5)$ & $6(8.2)$ & $25(71.4)$ & $12(34.2)$ & - & $9(36)$ & $5(20)$ & $3(12)$ \\
\hline Cryptococcus & $5(6.8)$ & $4(5.4)$ & - & $5(14.2)$ & $2(5.7)$ & - & $2(8)$ & - & - \\
\hline$T$ gondi ${ }^{2}$ & $1(1.3)$ & - & - & $1(2.8)$ & - & - & $12(48)$ & $1(4)$ & - \\
\hline Mycobacteria & $19(26)^{345}$ & - & - & $12(34.2)^{3}$ & $2(5.7)$ & - & $3(12)^{3}$ & - & - \\
\hline HSV & $2(2.7)$ & - & - & $11(31.4)$ & $1(2.8)$ & - & $\mathrm{nr}$ & - & - \\
\hline VZV & - & - & - & $4(11.4)$ & - & - & - & - & - \\
\hline Candida spp & $23(31.5)$ & - & - & $14(40)$ & - & - & $\mathrm{nr}$ & _- & - \\
\hline Aspergillus & $2(2.7)$ & - & - & $2(5.7)$ & - & - & - & - & - \\
\hline Histoplasma & $1(1.3)$ & $1(1.3)$ & - & - & - & - & - & - & - \\
\hline Bacteria & $32(43.8)$ & $1(1.3)$ & - & $5(14.2)$ & - & - & $2(8)$ & - & - \\
\hline Nocardia & - & - & - & $1(2.8)$ & - & - & - & - & - \\
\hline Lymphoma & $10(13.6)^{6}$ & - & - & $1(2.8)^{7}$ & - & - & $2(8)^{8}$ & $1(4)$ & - \\
\hline Kaposi's sarcoma ${ }^{9}$ & $25(34.2)$ & $-^{10}$ & - & $\mathrm{nr}$ & - & $3(8.5)^{11}$ & $11(44)$ & - & - \\
\hline
\end{tabular}

$\mathrm{S}=$ systemic, $\mathrm{S}+\mathrm{E}=$ systemic + eye, $\mathrm{E}=$ eye only, $\mathrm{nr}=$ not reported,${ }^{1}$ including cerebral $\mathrm{CMV},{ }^{2}$ cerebral only, ${ }^{3} \mathrm{M}$ avium intracellulare, ${ }^{4} M$ kansasii, ${ }^{5} M$ tuberculosis, ${ }^{6}$ six cerebral and two systemic non-Hodgkin's lymphoma (NHL) + two Hodgkin's lymphoma (HL), ${ }^{7}$ one cerebral NHL, ${ }^{8}$ two cerebral and one systemic NHL, ${ }^{9}$ cutaneous and visceral, ${ }^{10}$ two cutaneous involvement of eyelids, ${ }^{11}$ conjunctival.

no prophylactic therapy, the incidence of Pneumocystis carinii choroiditis appears to be low, as shown by Morinelli's study, ${ }^{8}$ where only four out of $20(1.9 \%)$ AIDS patients examined post mortem had suffered from ocular pneumocystosis (data from systemic involvement not reported).

Uveitis in the absence of an identifiable pathogen was a feature of 32 eyes. The possibility that the affected patients had a directly HIV related inflammation ${ }^{19}{ }^{20}$ must be allowed. On the other hand, immunohistochemical staining for the p24 antigen was consistently negative. A response to circulating immune complexes is another possibility. The current study includes patients dying before the availability of protease inhibitor combination regimens, which are known to cause an ocular inflammatory syndrome characterised by uveitis and cystoid macular oedema, as a result of restored specific immune reactivity. Recently, HIV, but no other micro-organism, was isolated in cultures of aqueous or vitreous from eyes of patients with idiopathic HIV associated uveitis. In these patients, symptoms resolved promptly with systemic zidovudine, strongly suggesting that HIV could have been the causative agent for their ocular inflammation. ${ }^{21}$ Differently from Levinson et $a l,{ }^{22}$ we observed no associated retinal infiltrates in the eyes of patients with chronic uveitis of unknown origin.

American experience gives an incidence for Kaposi's sarcoma in $10-24 \%$ of AIDS cases, ${ }^{6}$ but in the 133 patients studied histologically in the combined British, French, and American series there were only three cases of ocular (conjunctival) involvement. Diagnosis can be difficult in the absence of histological confirmation due, as in one of our cases, to the possibility of confusion with a localised haemorrhage.

We found no instances of intraocular lymphoma in our series, although five $(6.8 \%)$ had a non-Hodgkin's lymphoma in the brain. De Girolami and colleagues described a single instance in association with a CNS tumour. ${ }^{3}$
Overall, however, the incidence of intraocular lymphoma in AIDS patients appears to be very low.

In our study we noted focal collections of cytoid bodies, the histological counterpart of cotton wool spots, in 10 eyes, three linked to CMV retinitis. As such they represented the outcome of focal retinal ischaemia and can reasonably be attributed to occlusion of a terminal retinal arteriole. Where they were associated with CMV infection vasculitis could be implicated. Those occurring as part of a retinal microvasculopathy in the absence of CMV or other demonstrable infection are less easily explained but proffered mechanisms include vascular occlusion by circulating immune complexes, increased blood viscosity secondary to raised plasma fibrinogen levels, or even a direct effect of HIV on the vascular endothelium. The retinal haemorrhages observed in four cases with CMV retinitis and two without could also be the outcome of vasculitis. Other clinically described features of retinal vasculopathy such as capillary closure, vascular attenuation, and microaneurysms are not readily recognised in conventional histological sections and their absence in our findings is not significant.

An unexpected and previously unreported finding was the calcification affecting 14 eyes. Calcification of the sclera and ciliary processes is common in elderly subjects, but it is difficult to implicate senescence in generally young AIDS population. Nevertheless, the pattern and character of the deposits in most instances closely resembled those associated with ageing and an accelerated degenerative process seems likely, although the stimulus is obscure. The three patients with substantial corneal deposits, however, are not explained on this basis since the cornea does not normally share the sclera's predisposition to age related calcification. Furthermore, should dystrophic calcification affect the cornea for whatever reason in non-AIDS individuals it primarily involves Bowman's zone and the superficial stroma, whereas in the AIDS patients it was mainly located in the deeper lamellae with sparing of 
Bowman's zone. The patients' routine investigations indicated normal plasma calcium and phosphate levels. Even stranger was the finding that a further 11 eyes contained calcium oxalate crystals, although it might be noted that oxalates can occasionally present in the lens and in relation to long standing retinal detachments in non-AIDS patients as a presumed degenerative phenomenon. Although two of the 24 patients with ocular calcification or oxalosis also had CMV retinitis, none had received foscarnet, which has a propensity to disturb calcium balance.

The authors wish to thank Mr Ian Rhodes for his technical support. Dr Pecorella was given a grant by the Istituto Superiore di Sanità (Rome, Italy).

1 Rauz S, Murray PI . Changing patterns of HIV-related ocular disease. Sex Transm Inf 1999;75:18-20.

2 Seregard S. Retinochoroiditis in the acquired immune deficiency syndrome. Findings in consecutive post-mortem examinations. Acta Ophthalmol 1994;72:223-8.

3 De Girolami U, Hénin D, Girard B, et al. Etude pathologique de l'oeil et du système nerveux central dans 25 cas de SIDA. Rev Neurol (Paris) 1989;145:819-28.

4 Jensen OA, Klinken L. Pathology of brain and eye in the acquired immune deficiency syndrome (AIDS). A comparison of lesions in a consecutive autopsy material. APMIS 1989;97:325-33.

5 Pepose JS, Holland GN, Nestor MS, et al. Acquired immune deficiency syndrome. Pathogenetic mechanisms of ocular deficiency syndrome. Pathogenetic mech

6 Shuler JD, Holland GN, Miles SA, et al. Kaposi sarcoma of the conjunctiva and eyelids associated with the acquired the conjunctiva and eyelids associated with the acquired
immunodeficiency syndrome. Arch Ophthalmol 1989;107: immunodefi.

7 Klintworth GK. Degenerations, depositions, and miscellaneous reactions of the ocular anterior segment. In: Garne $\mathrm{A}$ and Klintworth GK, eds. Pathobiology of ocular disease. A dynamic approach. 2nd ed. New York: Marcel Dekker, 1994:743-94.
8 Morinelli EN, Dugel PU, Lee $M$, et al. Opportunistic intraocular infections in AIDS. Trans Am Ophthalmol Soc intraocular infections in

9 Jabs DA, Green WR, Fox R, et al. Ocular manifestations of acquired immune deficiency syndrome. Ophthalmology 1989;96:1092-9.

10 Holland GN, Engstrom RE, Glasgow BJ, et al. Ocular toxoplasmosis in patients with the acquired immune deficiency syndrome. Am f Ophthalmol 1988;106:653-67.

11 Cohen DB, Glasgow BJ. Bilateral optic nerve cryptococcosis in sudden blindness in patients with acquired immune deficiency syndrome. Ophthalmology 1993;100:1689-94.

12 Schuman JS, Friedman AH. Retinal manifestations of the acquired immune deficiency syndrome (AIDS): cytomegalovirus, Candida albicans, cryptococcus, toxoplasmosis and Pneumocystis carinii. Trans Ophthalmol Soc UK 1983;103: 177-90.

13 Specht CS, Mitchell KT, Bauman AE, et al. Ocular histoplasmosis with retinitis in a patient with acquired mmune deficiency syndrome. Ophthalmology 1991;98: 1356-9.

14 Ebert EM, D'Amico DJ. Differential diagnosis of the retinal manifestations of acquired immunodeficiency syndrome. Sem Ophthalmol 1993;8:2-14.

15 Grossniklaus HE, Specht CS, Allaire G, et al. Toxoplasma gondii retinochoroiditis and optic neuritis in acquired immune deficiency syndrome. Ophthalmology 1990;97: 1342-6.

16 Croxatto JO, Mestre C, Puente S, et al. Nonreactive tuberculosis in a patient with acquired immune deficiency syndrome. Am f Ophthalmol 1986;102:659-60.

17 Whitcup SM, Fenton RM, Pluda JM, et al. Pneumocystis carinii and Mycobacterium avium-intracellulare infection of the choroid. Retina 1992;12:331-5.

18 Wong B, Gold JW, Brown AE, et al. Central nervous system toxoplasmosis in homosexual men and parenteral drug abusers. Ann Intern Med 1984;100:36-42.

19 Farrell PL, Heinemann MH, Roberts CW, et al. Response of human immunodeficiency virus-associated uveitis to zidovudine. Am f Ophthalmol 1988;106:7-10.

20 O'Hara MA, Raphael SA, Nelson LB. Isolated anterior uveitis in a child with acquired immunodeficiency synuveitis in a child with acquired imm
drome. Ann Ophthalmol 1991;23:71-3.

21 Rosberger DF, Heinemann M, Friedberg D, et al. Uveitis associated with human immune deficiency virus infection. Am f Ophthalmol 1998;125:301-5.

22 Levinson RD, Vann R, Davis JL, et al. Chronic multifocal retinal infiltrates in patients infected with HIV. Am f Ophthalmol 1998;125:312-24. 